\title{
Regulation and Function of Collagen-specific Molecular Chaperone, HSP47
}

\author{
Kazuhiro Nagata* and Nobuko Hosokawa \\ Department of Cell Biology, Chest Disease Research Institute, Kyoto University, Sakyo-ku, Kyoto 606-01, \\ Japan
}

Proteins destined for secretion, or for incorporation into membranes, enter the endoplasmic reticulum (ER) co-translationally, where they fold and assemble into their active conformations. The ER contains several ER-resident proteins that are termed "molecular chaperones" or "folding enzymes". Molecular chaperones, such as HSP70 and GroEL, are involved in the processing and maturation of secretory and membrane proteins; they are thought to associate with folding intermediates or misfolded proteins to prevent nonproductive side reactions such as irreversible aggregation, and they may accelerate the slower steps in the folding process $(1,2)$.

Within the ER are enzymes that form disulfide bonds, such as protein disulfide isomerase (PDI) and peptidylprolyl isomerase (PPIase), and chaperone proteins, including the glucose-regulated proteins GRP94, GRP78/BiP (immunoglobulin heavy chain-binding protein), calnexin (an ER-membrane glycoprotein) and calreticulin (an ER luminal protein) $(3,4)$. Of these proteins, the folding enzymes, PDI and PPIase, are involved in the disulfide bond formation in the nascent polypeptides in the ER. The sequential formation of disulfide bonds is required for normal folding and for subsequent transport to the Golgi apparatus and beyond. Certain chaperone proteins such as GRP78 and GRP94 are thought to facilitate translocation into the ER and then to assist in folding, oligomeric assembly and sorting in the ER. These soluble ER-resident proteins have the sequence Lys-Asp-Glu-Leu (KDEL) at their carboxyl terminus, which acts as an ER-retention signal (5). Some ER-resident type I transmembrane proteins such as calnexin contain an ER-targeting motif consisting of two lysine residues at the carboxyl terminus (6).

HSP47 is another ER resident protein, first identified as the major collagen-binding heat-inducible glycoprotein in fibroblasts (7). It is characterized by its substrate specificity for collagen, and plays a major role in collagen processing and quality control under stress conditions, by preventing the secretion of procollagen with abnormal conformation (8).

\footnotetext{
* To whom correspondence should be addressed.

Tel: 81-75-751-3848, FAX: 81-75-751-4645

e-mail:nagata@chest.kyoto-u.ac.jp
}

\section{Association of HSP47 with Procollagen}

HSP47, which has a molecular mass of approximately $47 \mathrm{kDa}$, was first identified in chick embryo fibroblasts as a collagen-binding glycoprotein whose expression was down-regulated after malignant transformation with Rous sarcoma virus $(7,9)$. After its initial identification, cDNA cloning revealed that HSP47 belongs to the serpin (serine protease inhibitor) superfamily, which includes $\alpha 1$-antitrypsin, $\alpha 1$-antichymotrypsin, antithrombin III and ovalbumin (10). HSP47 has no protease inhibitor activity. HSP47 has a signal sequence at its amino terminus and an ER-retention signal sequence, RDEL (Arg-Asp-Glu-Leu) at its carboxyl terminus. A mutant HSP47 which lacks the RDEL sequence was shown not to be retained in the ER any more, but to be secreted out of the cells (11). As expected from this molecular nature, HSP47 was shown to be localized in the ER by the observation of the cells by immunostaining and immuno-electron microscopy (12). The analysis of carbohydrate indicated that HSP47 was glycosylated at two gylcosylation sites, and that both of them were high mannose type, which also supports the view that HSP47 is an ER-resident glycoprotein $(11,13)$. Similar collagen-binding proteins were also reported as colligin and gp46 in the mouse teratocarcinoma cell-line F9 and rat L6 myoblasts, respectively, and were later established to be identical to HSP47 $(14,15)$.

Pulse label/chase experiments combined with crosslinking and immunoprecipitation have revealed that HSP47 transiently binds to newly synthesized procollagen in the ER (11). Immediately after the $\alpha$ chains of procollagen enter the ER, HSP47 binds to these nascent $\alpha$ chains. When polysome fractions were isolated and immunoprecipitated with anti-HSP47 antibody, nascent procollagen chains of various lengths were coprecipitated with HSP47, which again suggested that HSP47 binds to nascent polypeptides of procollagen $(16,17)$. HSP47 also binds to a triple helix form of procollagen which was examined by the resistance of immunoprecipitated collagen chains against trypsin digestion (11). In vitro binding assay using purified mature collagen also indicated that HSP47 binds to the triple helix form of mature collagen (18). 


\section{Dissociation of HSP47 from Procollagen}

To assess the site where HSP47 dissociates from procollagen in the central secretory pathway, we treated the cells with various inhibitors for secretion, and then analyzed the dissociation of HSP47 from procollagen by pulse label/chase experiment. Confocal microscopic observation after simultaneous staining of the cells with anti-HSP47 and anti-collagen antibodies was also used for this approach. $\alpha, \alpha^{\prime}$-dipyridyl, an iron-chelating agent, is known to inhibit the proryl and lysyl hydroxlyation of procollagen, resulting in the prevention of triple helix formation and of secretion of procollagen out of the cells $(19,20)$. Brefeldin A is known to release coat proteins and ADP ribosylation factor from the Golgi stack, and thus inhibits the transport of secretory proteins from the ER to the Golgi (21). Monensin is an ionophore for $\mathrm{Na}^{+}, \mathrm{K}^{+}$and protons, and is known to inhibit intracellular transport within the Golgi complex, preferentially between the cis- or medial-Golgi and trans-Golgi compartment (22).
In the normal fibroblasts, newly synthesized procollagen is secreted within $20 \mathrm{~min}$, from which HSP47 dissociates in a little less than $20 \mathrm{~min}$. However, when cells were treated with $\alpha, \alpha^{\prime}$-dipyridyl or brefeldin $\mathrm{A}$, the secretion of procollagen was completely inhibited (11, 19). The pulse/chase and immunoprecipitation experiments revealed that HSP47 bound to newly synthesized procollagen was not dissociated from it for up to $2 \mathrm{~h}$ (Fig. 1). Immunostaining observation using confocal microscopy showed that both HSP47 and procollagen colocalized in the ER and in the intermediate compartment between the ER and the Golgi in the $\alpha, \alpha^{\prime}$-dipyridyl-treated and brefeldin A-treated cells, respectively (Fig. 2) (11). These results suggested that HSP47 does not dissociate from procollagen either in the ER or in the intermediate compartment. When the cells were treated with monensin, the dissociation of HSP47 from procollagen occurred within $15 \mathrm{~min}$ after pulse labeling, whereas the secretion of procollagen out of the cells was totally inihibited (11). Double staining of HSP47 and

A

\begin{tabular}{|c|c|c|}
\hline treat & $\begin{array}{ll}n & 0\end{array}$ & $\alpha, \alpha^{\prime}$ \\
\hline chase & 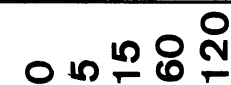 & 0 ㄴ \\
\hline KDa & & 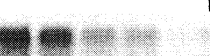 \\
\hline
\end{tabular}

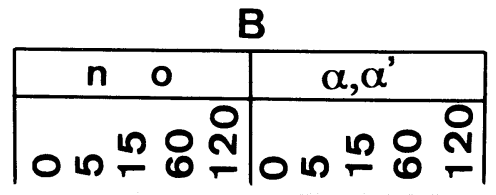

$94-$

67
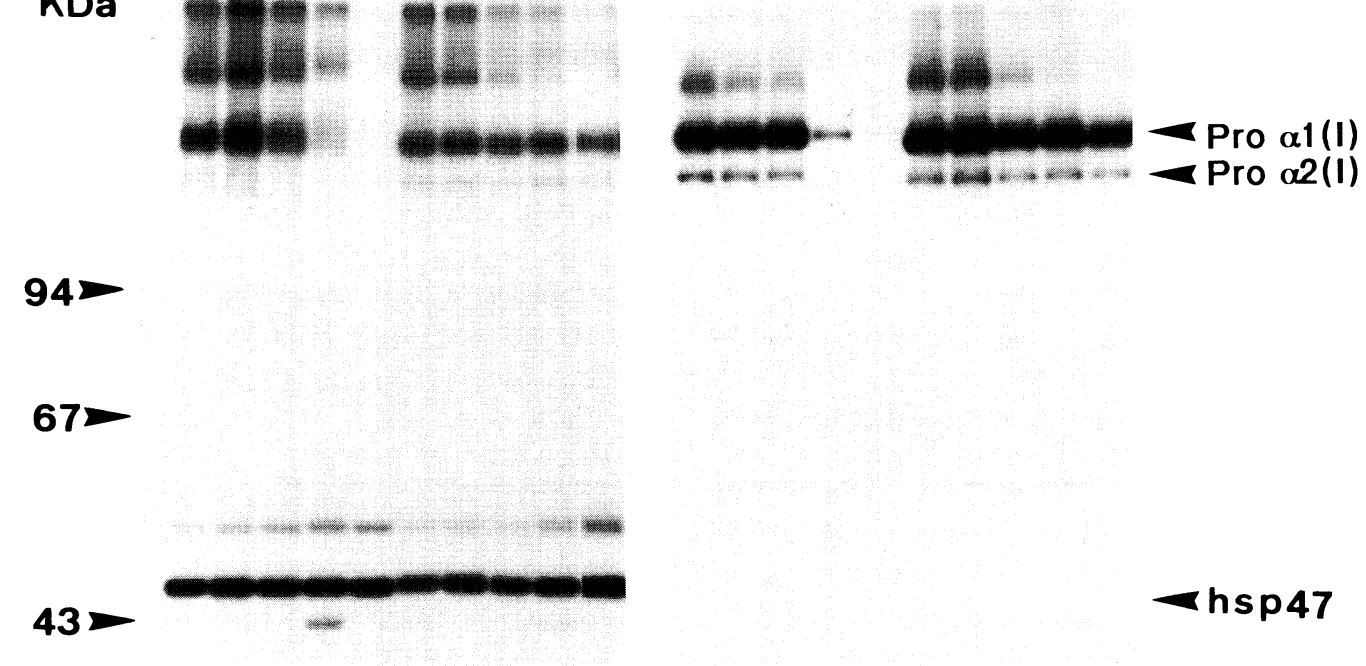

- hsp47

Fig. 1. Effect of $\alpha, \alpha^{\prime}$-dipyridyl on the kinetics of total procollagen and procollagen coprecipitated with HSP47. Cells were pulse labeled for 10 min with $\left.{ }^{35} \mathrm{~S}\right]$ methionine and chased in the medium containing excess methionine in the presence (indicated on the top of the panel as $\left.\alpha, \alpha^{\prime}\right)$ or absence (indicated as no) of $\alpha, \alpha^{\prime}$-dipyridyl for various periods. Immune complexes precipitated with anti-HSP47 antibody (A) or anti-collagen serum (B) were applied to SDS-8\% polyacrylamide gel. The numbers on the top of the each lane show the chase periods in min. 


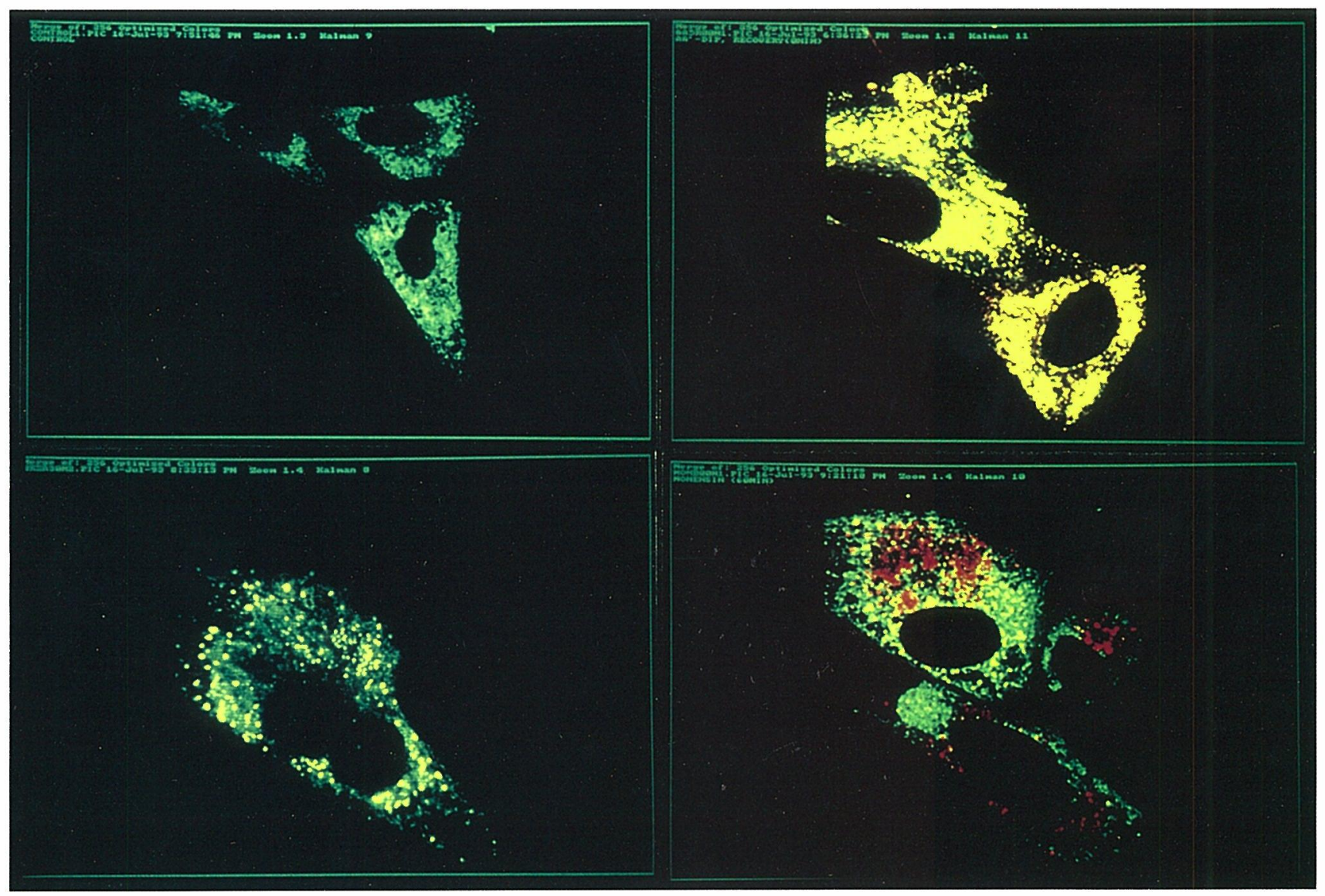

Fig. 2. Localization of HSP47 and procollagen in cells (chick embryo fibroblasts) treated with ascorbate (upper left panel), $\alpha, \alpha^{\prime}$-dipyridyl (upper right panel), brefeldin A (lower left panel) and monensin (lower right panel). The cells were fixed, permeabilized, and double stained with the rat anti-HSP47 monoclonal antibody and the rabbit anti-type I collagen antiserum. Visualization was performed with FITC-conjugated goat anti-rat IgG for HSP47, and with rhodamine-conjugated goat anti-rabbit IgG for procollagen. Images were obtained with a scanning laser confocal microscope. The localizations of HSP47 and procollagen were shown by green and red color, respectively, and the colocalization of both proteins was by yellow color.

procollagen with specific antibodies also supported the above result: Golgi area was stained with anti-collagen antibody whereas the ER-staining pattern was observed with anti-HSP47 antibody (Fig. 2). These results suggested that HSP47 dissociated from the procollagen when it entered the Golgi compartment. Thus, HSP47 binds to the procollagen immediately after it enters the ER and dissociates from it in the cis-Golgi network (8, $11)$.

\section{HSP 47 as a Molecular Chaperone}

As is well known, procollagen $\alpha$ chains begin to form a trimer at the $\mathrm{C}$-propeptide domain via the formation of disulfide bonds between each C-propeptide chain in the ER. Once $\alpha$ chains make trimers at the C-terminus, the triple helix formation proceeds from the C-propeptide toward N-propeptide. This implies that nascent polypeptide $\alpha$ chains should be maintained unfolded un- til they make trimers, otherwise triple helix formation would be prevented. HSP47 may prevent nascent $\alpha$ chains of procollagen from being folded or from making random aggregations in the ER. These possible functions of HSP 47 may be attributed to those of molecular chaperones.

When cells were heat shocked or treated with $\alpha, \alpha^{\prime}$ dipyridyl, HSP47 did not dissociate from procollagen during the chase period up to $2 \mathrm{~h}$ whereas the dissociation occurred within $15 \mathrm{~min}$ in non-treated control cells $(11,19)$. These results suggest that the function of HSP47 under stress conditions may be similar to a quality control mechanism like BiP/GRP78 (8).

\section{Correlational Expression of HSP47 with Collagen Genes}

As mentioned above, HSP47 is thought to be a molecular chaperone which is specific to collagen. In addition 
Table I. Correlation of Hsp47 synthesis to collagen Synthesis.

\begin{tabular}{|c|c|c|c|c|c|c|}
\hline \multirow{2}{*}{ Cell/tissue type } & \multirow{2}{*}{ Cell line/type } & \multirow{2}{*}{ Hsp47 } & \multicolumn{4}{|c|}{ Collagen (type) } \\
\hline & & & $\mathrm{I}$ & II & III & IV \\
\hline Fibroblast & $\begin{array}{l}\text { CEF } \\
\text { RSV-transformed CEF } \\
\text { BALB/3T3 } \\
\text { SV40-transformed 3T3 } \\
\text { NIH3T3 } \\
\text { c-Ha-Ras-transformed 3T3 }\end{array}$ & $\begin{array}{l}\text { H } \\
+ \\
+1 \\
+ \\
+ \\
+\end{array}$ & $\begin{array}{l}\text { m } \\
+ \\
\text { m } \\
+ \\
+ \\
+\end{array}$ & & & \\
\hline Adipocyte & $\begin{array}{l}\text { 3T3-L1 } \\
\text { Differentiated 3T3-L1 }\end{array}$ & $\begin{array}{l}\text { H } \\
H\end{array}$ & $\begin{array}{l}\text { m } \\
H\end{array}$ & & & $\begin{array}{l}+ \\
+\end{array}$ \\
\hline Myelocyte & M1 & - & - & - & - & - \\
\hline Neurocyte & $\mathrm{PC} 12$ & - & - & - & - & - \\
\hline Embryonic carcinoma & $\begin{array}{l}\text { F9 } \\
\text { F9 } \\
\text { F9 }^{\mathrm{b}}\end{array}$ & $\begin{array}{l} \pm \\
H \\
H\end{array}$ & & & & $\begin{array}{l} \pm \\
H \\
H\end{array}$ \\
\hline Hepatic tissue & $\begin{array}{l}\text { Hepatocyte } \\
\text { Itoh cells } \\
\text { Fibrosis in rat liver }\end{array}$ & $\begin{array}{l}- \\
H \\
H\end{array}$ & - & - & $\begin{array}{l}- \\
H \\
H\end{array}$ & - \\
\hline Bone & Osteoblasts & H & m & & & \\
\hline Cartilage & Chondrocytes & H & & H & & \\
\hline Teeth & Odontoblasts & $H$ & H & & & \\
\hline
\end{tabular}

a Treated with retinoic acid.

b Treated with retinoic acid and dibutyryl cAMP.

to this functional relevance with collagen, the expression of HSP47 in various cells is closely correlated with that of collagen. The synthesis of type I collagen is at a high level in chick embryo fibroblasts as reported previously. In these fibroblasts, the level of HSP47 synthesis is also high. When the cells were transformed with Rous sarcoma virus, the synthesis of both type I collagen and HSP47 were much decreased (7). Simultaneous reduction in the synthesis of these two proteins were similarly observed after malignant transformation of mouse fibroblastic cells with Simian virus 40 or activated c-Haras oncogene (Table I) $(23,24)$.

Interestingly, HSP47 is actively synthesized in the cells producing high amounts of any type of collagen while it is hardly detected in the cells which do not synthesize detectable level of collagens. For example, HSP47 synthesis was not detected at all in mouse myeloid leukemic cell line, M1, or mouse pheochromocytoma cell line, PC12, where collagen synthesis was not detected either $(8,24)$. In mouse teratocarcinoma cell line, F9, neither HSP47 or types I to IV collagen was detected in an undifferentiated condition. However, the synthesis of both HSP47 and type IV collagen were markedly increased after the differentiation of F9 cells by the treatment with retinoic acid or retinoic acid plus dibutylyc cyclic AMP (Table I) $(14,25)$. Thus, the expression of HSP47 is likely co-regulated with that of collagens.

\section{Induction of HSP47 during the Progression of Liver Fi- brosis}

To examine whether such a correlation in the synthesis between HSP47 and collagen is also observed in the tissues under pathophysiological conditions, we analyzed the expression of HSP47 and collagen in the experimental liver fibrosis model of rats (26). Rats were orally administered carbon tertrachloride (CTC) for 2 to 12 weeks. The mRNAs both for types I and III collagens and for HSP47 were hardly detectable in the liver of control rats. After two weeks of CTC administration, marked accumulation of mRNAs was observed in Northern blot analysis not only for types I and III collagens but also for HSP47. High levels of mRNA for these proteins were maintained up to 12 weeks in the liver of CTC-administered rats. From in situ hybridization analysis with the probes for HSP47 and type I collagen and immunostaining with the antibody against desmin, a marker for Itoh cells in the liver, we concluded that the HSP47 producing cells in the liver during the progression of liver fibrosis are the Itoh cells, which has been known to be the collagen-producing cells in the liver (26). Coexpression of the genes encoding both HSP47 and collagen is also reported in the development of murine femurs and molars and various chick tissues $(27,28)$.

\section{Conclusion}

HSP47 can be thought of as a substrate-specific molecular chaperone that only interacts with certain procol- 
lagens within the cells. Although HSP47 was reported to bind fetuin in vitro (29), immunoprecipitation shows that the major secretory protein co-precipitated with HSP47 is procollagen in the fibroblasts. A more detailed examination of whether HSP47 binds to other secretory or membrane proteins in the ER is still required. It will also be important to examine whether these substrate-specific molecular chaperones work with other general chaperones in the ER, such as BiP and calnexin. HSP47 is reported to associate with BiP, PDI (19) and cyclophilin B (30), but it is not clear whether this association has biological implications for its chaperone function. Finally, it would be one of the most interesting questions to determine how the expression of HSP47 is co-regulated with that of collagen. Recently, we have got the results that the reduction of HSP47 synthesis by the transfection of antisense RNA for HSP47 into the mouse fibroblasts causes the inhibition of type I collagen synthesis. This result also suggests the mechanism of co-regulation of HSP47 and collagen, which should be disclosed in near future.

\section{REFERENCES}

1. HaRtL, F.U. 1996. Molecular chaperones in cellular protein folding. Nature, 381: 571-579.

2. Morimoto, R.I., Tissieres, A., and Georgopoulos, C. eds. 1994. "The Biology of Heat Shock Proteins and Molecular Chaperones", Cold Spring Harbor Laboratory Press, pp. 610.

3. Gething, M.J. and Sambrook, J. 1992. Protein folding in the cell. Nature, 355: 33-45.

4. Bergeron, J.J., Brenner, M.B., Thomas, D.Y., and Williams, D.B. 1994. Calnexin: a membrane-bound chaperone of the endoplasmic reticulum. Trends Biochem. Sci., 19: 124-128.

5. Munro, S. and Pelham, H.R. 1987. A C-terminal signal prevents secretion of luminal ER proteins. Cell, 48: 899-907.

6. Nilsson, T., JACkson, M., and Peterson, P.A. 1989. Short cytoplasmic sequences serve as retention signals for transmembrane proteins in the endoplasmic reticulum. Cell, 58: 707-718.

7. Nagata, K., SAGA, S., and Yamada, K.M. 1986. A major collagen-binding protein of chick embryo fibroblasts is a novel heat shock protein. J. Cell Biol., 103: 223-229.

8. NAGATA, K. 1996. HSP47: a collagen-specific molecular chaperone. Trends Biochem. Sci., 21: 23-26.

9. Nagata, K. and Yamada, K.M. 1986. Phosphorylation and transformation sensitivity of a major collagen-binding protein of fibroblasts. J. Biol. Chem., 261: 7531-7536.

10. Hirayoshi, K., Kudo, H., Takechi, H., NakaI, A., Iwamatsu, A., Yamada, K.M., and Nagata, K. 1991. HSP47: a tissuespecific, transformation-sensitive, collagen-binding heat shock protein of chicken embryo fibroblasts. Mol. Cell Biol., 11: 4036-4044.

11. Satoh, M., Hirayoshi, K., Yokota, S.-I., Hosokawa, N., and NAGATA, K. 1996. Intracellular interaction of collagen-specific stress protein HSP47 with newly synthesized procollagen. $J$. Cell Biol., 133: 469-483.

12. Saga, S., Nagata, K., Chen, W.T., and Yamada, K.M. 1987. pH-dependent function, purification, and intracellular location of a major collagen-binding glycoprotein. J. Cell Biol., 105: 517-527.

13. Hughes, R.C., Taylor, A., Sage, H., and Hogan, B.L. 1987. Distinct patterns of glycosylation of colligin, a collagenbinding glycoprotein, and SPARC (osteonectin), a secreted $\mathrm{Ca}^{2+}$-binding glycoprotein. Evidence for the localisation of colligin in the endoplasmic reticulum. Eur. J. Biochem., 163: 5765.

14. Kurkinen, M., Tayler, A., Garrels, J.I., and Hogan, B.L. 1984. Cell surface-associated proteins which bind native type IV collagen or gelatin. J. Biol. Chem., 259: 5915-5922.

15. Cates, G.A., Nandan, D., Brickenden, A.M., and Sanwal, B.D. 1987. Differentiation defective mutants of skeletal myoblasts altered in a gelatin-binding glycoprotein. Biochem. Cell Biol., 65: 767-775.

16. SAUK, J.J., Smith, T., Norris, K., and Ferreira, L. 1994. Hsp47 and the translation-translocation machinery cooperate in the production of alpha 1 (I) chains of type I procollagen. $J$. Biol. Chem., 269: 3941-3946.

17. Hu, G., Gura, T., Sabsay, B., Sauk, J., Dixit, S.N., and Veis, A. 1995. Endoplasmic reticulum protein HSP47 binds specifically to the $\mathrm{N}$-terminal globular domain of the amino-propeptide of the procollagen I $\alpha_{1}$ (I)-chain. J. Cell Biochem., 59: 350367.

18. Natsume, T., Koide, T., Yokota, S., Hirayoshi, K., and NAGATA, K. 1994. Interactions between collagen-binding stress protein HSP47 and collagen. Analysis of kinetic parameters by surface plasmon resonance biosensor. J. Biol. Chem., 269: 31224-31228

19. NaKaI, A., Satoh, M., Hirayoshi, K., and Nagata, K. 1992. Involvement of the stress protein HSP47 in procollagen processing in the endoplasmic reticulum. J. Cell Biol., 117: 903-914.

20. KaO, W.W., Prockop, D.J., and Berg, R.A. 1979. Kinetics for the secretion of nonhelical procollagen by freshly isolated tendon cells. J. Biol. Chem., 254: 2234-2243.

21. Lippincott-Schwartz, J., Yuan, L.C., Bonifacino, J.S., and KLAUSNER, R.D. 1989. Rapid redistribution of Golgi proteins into the ER in cells treated with brefeldin A: evidence for membrane cycling from Golgi to ER. Cell, 56: 801-813.

22. TaRtakofF, A.M. 1983. Perturbation of vesicular traffic with the carboxylic ionophore monensin. Cell, 32: 1026-1028.

23. NAKaI, A., Hirayoshi, K., and Nagata, K. 1990. Transformation of BALB/3T3 cells by simian virus 40 causes a decreased synthesis of a collagen-binding heat-shock protein (hsp47). $J$. Biol. Chem., 265: 992-999.

24. Nagata, K., Nakai, A., Hosokawa, N., Kudo, H., Takechi, H., SATOH, M., and Hirayoshi, K. 1991. Interaction of HSP47 with newly synthesized procollagen, and regulation of HSP expression. In Heat Shock. B. Maresca and S. Lindquist, editors. Spring-Verlag, New York, 105-110.

25. TaKechi, H., Hirayoshi, K., NaKai, A., Kudo, H., Saga, S., and NAGATA, K. 1992. Molecular cloning of a mouse 47-kDa heat-shock protein (HSP47), a collagen-binding stress protein, and its expression during the differentiation of $\mathrm{F} 9$ teratocarcinoma cells. Eur. J. Biochem., 206: 323-329.

26. Masuda, H., Fukumoto, M., Hirayoshi, K., and Nagata, K. 1994. Coexpression of the collagen-binding stress protein HSP47 gene and the alpha 1(I) and alpha 1(III) collagen genes in carbon tetrachloride-induced rat liver fibrosis. J. Clin. Invest., 94: 2481-2488.

27. Shroff, B., Smith, T., Norris, K., Pileggi, R., and Sauk, J.J. 1993. Hsp47 is localized to regions of type I collagen production in developing murine femurs and molars. Connect. Tissue. 
Res., 29: 273-286.

28. Miyaishi, O., Sakata, K., Matsuyama, M., and Saga, S. 1992. Distribution of the collagen binding heat-shock protein in chicken tissues. J. Histochem. Cytochem., 40: 1021-1029.

29. Nakai, A., Hirayoshi, K., Saga, S., Yamada, K.M., and NAGATA, K. 1989. The transformation-sensitive heat shock protein (hsp47) binds specifically to Fetuin. Biochem. Biophys. Res. Commun., 164: 259-264.
30. Smith, T., Ferreira, L.R., Hebert, C., Norris, K., and SaUK, J.J. 1995. HSP47 and cyclophilin B traverse the endoplasmic reticulum with procollagen into pre-Golgi intermediate vesicles. A role for HSP47 and cyclophilin B in the export of procollagen from the endoplasmic reticulum. J. Biol. Chem., 270: 1832318328. 